\title{
Intelligent Controller Design of the Vehicle Sonar Tracking Checker Fault and Repair of High Voltage Transmission Line
}

\author{
Saja Raad Abdulbari Hani ${ }^{1}$, Dr. Abdullah Al Gizi ${ }^{2}$ \\ \{saja.alhilfi@gmail.com ${ }^{1}$, abdullah.algizi@stu.edu.iq ${ }^{2}$ \} \\ Engineering Technical College/Basra, Southern Technical University
}

\begin{abstract}
Inspection of high-voltage transmission lines is a dangerous and timeconsuming activity that requires specially trained technicians to work tens of meters above the ground and near-live lines carrying thousands of volts. This paper presents the development of a teleoperated Intelligent Controller Vehicle Sonar Tracking Checker designed by use UAV in the collection of data power lines for preventive maintenance of high-voltage lines. The Vehicle Sonar Tracking Checker was designed with mobility in mind, so those cable spacers, suspension clamps, and other obstacles, which so far have prevented the inspection of high-voltage lines, will not be prevented the inspection of the lines. The safe operation of the transmission line and its components, on the other hand, necessitates periodical inspections to detect corrosion and other climatic and mechanical problems and flaws. The necessity for rapid and robust algorithms for the interpretation of photos or video obtained by drones during inspections has increased as the usage of unmanned autonomous vehicles (UAVs) for ecological observation grows. use UAV in the collection of power lines data and for analysis defects, various algorithms are used from image processing with MATLAB and python.
\end{abstract}

Keywords: overhead power line, open-source computer vision, image processing, UAV

\section{Introduction}

Creating modern shrewd cities requires to coordinate a few savvy arrangements different areas together and putting all these solutions into home in a perfect way" most perfect way. As portion of this global scene, numerous things about were distributed within the final few long time presenting the modern term "smart grids" into our life. This unused era of control frameworks ought to be able to keep an ideal execution for all stages of the control era counting the final organize of control utilization and the exchange stages. This exceedingly wanted execution requires a part of imaginative thoughts. As a result, new clever self-maintenance scenarios can provide a robust mending execution while reducing the associated time and expenses when compared to traditional upkeep tactics. Foot watch or helicopter-based exams are the most well-known methods of inspecting control lattice hardware[1]. Luis f. Luque-vega, Bernardino Castillo-toledo, and alexander loukianov cinvestav-ipn unidad guadalajara in [2] present an aerial robotic system for power line inspection implemented from scratch, including the flight control system, the payload for inspection system makes use of a colour camera and IR (infrared) camera to inspect the devices and components in power line corridors. The most common method is modest and generally ineffective. Helicopter-assisted assessments are faster, but because they are unable to wander more than a short distance from the control frameworks, their visual frameworks or any other sensor will be unable to provide reliable estimates 
following that, an unmanned ethereal vehicle-based robot plan is proposed here to investigate and implement a few critical upkeep techniques for various control framework components. The paper [3] shows the defect detection result of an electrical insulator with simulated crack. It gives the edge detection of original ceramic electrical insulators without crack and defects ceramic electrical insulators with crack. W. Jiang, f. Wenkai, and 1. Qianru in [4] describes a UAV with a multi-path ground coordinating system based on $2 \mathrm{~d}$ plane lase radar. Both finals ponder were enthralled by the encirclement of the exchange lines, but they were unconcerned about the condition of the lines themselves. A new algorithmic approach for bunch drops is in[5]. Fault detection and location determination are based both on spatial morphological glass and ceramic insulators. Features proposed. Features proposed. The solution given can be applied to recognize UAV captured aerial photos with complicated background spatial-based glass and ceramic insulators features. The target area can then be morphologically determined developed to emphasize the position of the fault. In consequence, the fault insulators can be located based on the identity and features of the space. M. Pech, j. Vrchota, and j. Bednár [6] used the kalman channel and the Hough change to monitor the control lines based on a video arrangement stream. A climbing robot has been proposed by a few thinkers, as shown in, this robot can walk along cables to induce a close examination of the state of electrical components and to achieve the needed results. The study is organized as follows: within another section, we examine the many types of sensors and actuators that are required to conduct a few specific electrical lines support methods. The paper points to audit the current writing concerning prescient upkeep and shrewdly sensors in savvy factories. The projection is analysed utilizing the facilitates and distinguishing proof focuses for recognizing the object[7]. Siddiqui, Zahid Ali park, unsung in [8] proposed method to detect the fault of splits and puncture in polymer insulator by using ellipse detection algorithm and then apply the splits algorithm on individual caps, also proposed method to detect the broken or missing ceramic disk by colour clustering and morphological operations. In their research paper [9] they developed and implemented powerful algorithms for artificial vision, distance calculation, and hot spot detection[9].this paper[10] talks about the utilize of multi-agent frameworks as a reasonable arrangement to address this challenge by analysing their benefits when applying them to the field of savvy lattices and looking over existing works and activities. The foremost important specialized details of this robot are delineated within the third segment. Within the fourth section, a few fundamental computer vision algorithms are used to need a few estimations and readings from the flying robot's environment. Within the fifth segment, a modern locale proposition method that depends on a discrete wavelet change (dwt)[11]. Also, this work uses a flying robot named the butterfly to repair damaged or weakened exchange or dispersion control links, especially in inaccessible areas or high mountains. This robot is also required to continuously inspect all components of a control system using various types of visual and thermal sensors to rapidly identify any issues that may jeopardize its functionality. There are many difficulties you encounter when using drone such as the sales are expected 6 million and specific drones can used more one sensors[12].this article, we used a vehicle, as shown in fig. 1, to scan a power transmission line and distinguish between the line conductor and insulator in order to repair damaged or debilitated exchange or dispersion control lines, particularly in difficult places or high mountains. The subject of high-power lines fault detection using drones is an interesting and important subject to be considered due to risk, cost time consuming and difficulties associated with geographical areas of line towers. 


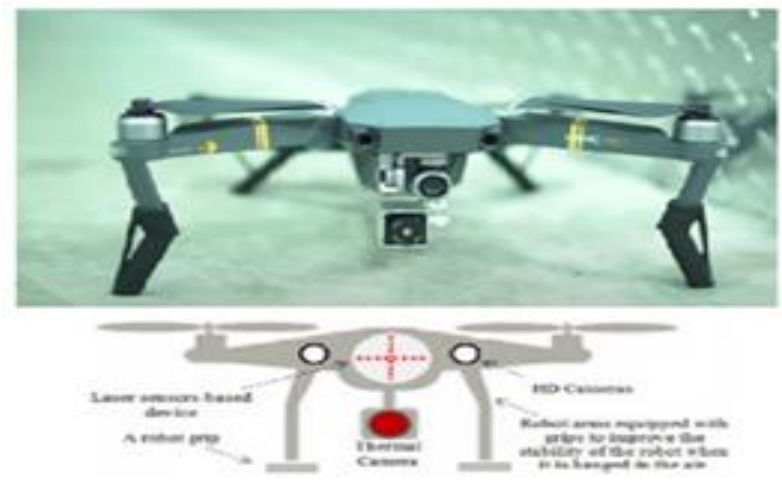

Fig.1. A view of the vehicle robot from the front[11].

\section{Methodology of The Proposed System}

The drone will be equipped with a thermal camera reader, which will take warm images that will be useful for inspecting cables, electrical connections, switches, and circuit breakers across the area. The HD cameras worked depends on takes pictures are valuable to check cables. Our transmission line examination system is consisting of a thermal camera, an HD camera, and a drone. This system is examining all parts of the transmission line and taking close-up pictures in which the status of each transmission line part are examined, for example, towers, insulators, transmission poles, insulators, borselen beacon, sag adjusters, and the external appearance of the outer surface of the conveyor line (the skin layer) and checked for fracture, smash and corrosion with the external perimeter of the transmission line and store these images until the external processors are images MATLAB or python. The main aim of using this system is to reduce monitoring time and costs without declining quality, to enhance employee safety during the inspection. Fig. 1 showing a view of the vehicle robot from the front that is crucial for the secure transfer of power between a network station and a local distribution station. These components can withstand extreme weather (direct sunlight, strong winds, snow, rain, and so on) as well as severe mechanical tension and high temperature due to high voltage. The proposed techniques are a combination of image MATLAB processing and python techniques.

\subsection{Thermal camera}

The Stefan-Boltzmann law, which quantifies the power emitted from an object as an item of the Stefan-Boltzmann consistent, and the thermodynamic temperature of that question within the power of 4 , is the foundation of thermal imaging. The most common idea is to create a picture using infrared radiations emitted by a question that is proportional to its temperature. Warm cameras differentiate radiations in the long wave of range extend $(8-14 \mathrm{~lm})$ or the mid-wave of range extend $(3-5 \mathrm{~lm})$ for the most part. Warm images are useful for inspecting cables, electrical connections, switches, and circuit breakers, and identifying a variety of control system flaws such as voltage switch oxidation, overheated or incorrectly secured connections, and the effects of collapsed protection. Insulator heating is not due to corona activity but there may be heating if leakage currents are flowing either on the surface insulator or through the heart. Heating inside 
the heart may indicate a damaged core during leakage current activity on the insulator surface may indicate damage to sheds and insulating sheath or perhaps associated with small partial brackets (dry tape brackets) on surface appearance[13]. As a rule, when the control framework changes any half-value of available vitality into extraneous calamities (warm), its capability decreases. To find the defect component of the overhead power line from IR image using the mean of the temperatures in each hot spot is obtained for comparison. If the temperature obtained from the thermal map hot spot is the same, then there is no fault and the temperature of all component's same degree. If there is a difference in hot spot temperature for the same component, then there is a fault that must be repaired or replaced. We used a method for analysing thermal images including convert colour images to grey images and apply k-means clustering unsupervised machine learning algorithm with $\mathrm{k}=18$ to segment different parts of an image using OpenCV in python, with otsu-threshold and dilation operation mask $(8,8)$ to highlight the hot spot region[14]. Fig 2 shows the algorithm steps where we can see two insulators in the infrared image, one defective and the other normal. The faulty insulator appears as the highlight and hotspot extraction region, while the normal insulator disappears in the hotspot region. the main aim is to reduce monitoring time and costs without declining quality, to enhance employee safety during the inspection. Transmission power lines, transmission shafts, insulators, balance beacons, and sag adjusters are important components of an electric transmission system. These components can withstand extreme weather (direct sunlight, strong wind, snow, rain, etc.) As well as high mechanical stress and temperature extremes due to high voltage. Traditional inspection methods include sending a skilled inspector up a power pole to look for electrical problems or managing the electrical health of goods by time-consuming, expensive, and dangerous experimental operations[1].
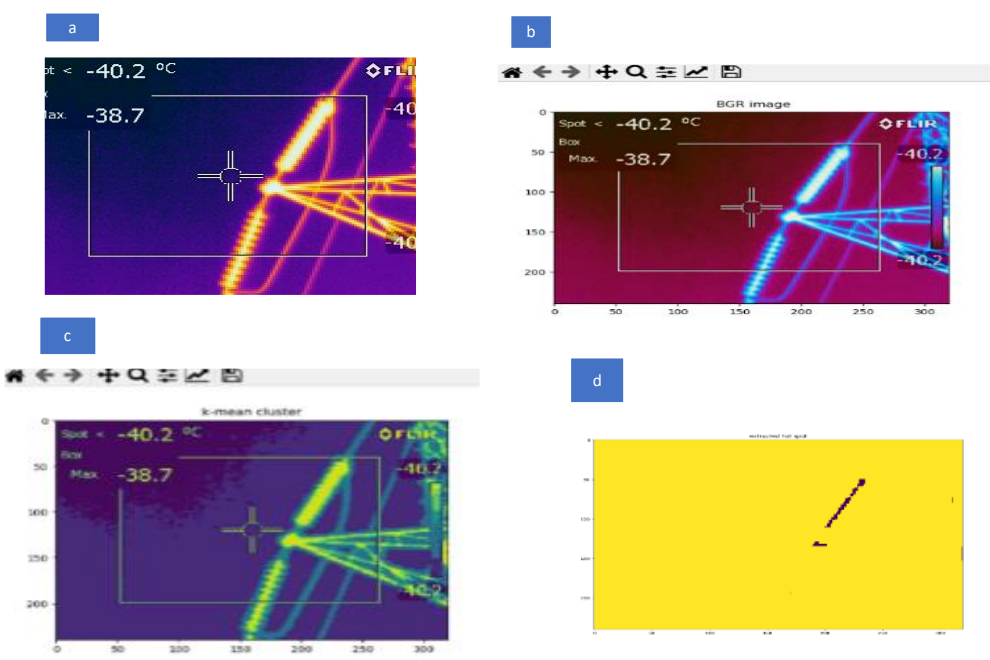

Fig.2. a) The thermal image shows two insulators, one defective and the other normal, b) Thermal image processing in BGR space, c) Thermal image after segmentation is applied, d) The result of the algorithm used to process the thermal image shows the hot spot of a defective high-temperature insulator. 


\subsection{Overhead Power Line HD IMAGES}

Many elements, such as attitudes of employees, terrain structure, and climate, can affect the traditional method of human inspection. The drone inspection can therefore increase overall efficiency and quality and guarantee the safe functioning and operation of the transmission line with faulty automatic insulator detection algorithm[15].because it can take a few aerial pictures from short distances for suspected or harmed control components, and because it can stay floating within the discussion about the same component as shown in fig.3.the quadcopter becomes a capable instrument to precisely look at any control electrical framework by using progressive pictures taken from various points (principal component analysis), as well as the proposal techniques are a combination between the image MATLAB processing and python techniques. This proposed method is based on the use of the drone in examining the network of high-voltage transmission lines by taking high-resolution images with the HD camera. These images are processed in two ways, either by using MATLAB through the image processor, analysing and comparing them with the standard to determine the quality of this conductor or insulator. The third way is to examine the line or network with the naked eye through filming a video in which all parts of the line are examined, including insulators, lines, connectors, and suspension tools from fracture, shattering, corrosion, and oxidation. For example, the algorithm for broken insulators disk defect in MATLAB is done by converting an RGB image to Gray image, apply canny edge detection, show and compare defect region and the normal region as shown in figs.7,8,9.

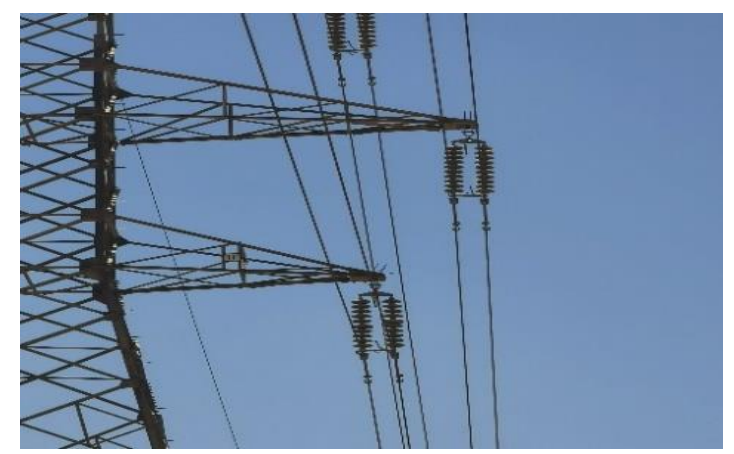

Fig.3. Overhead power line visible image.

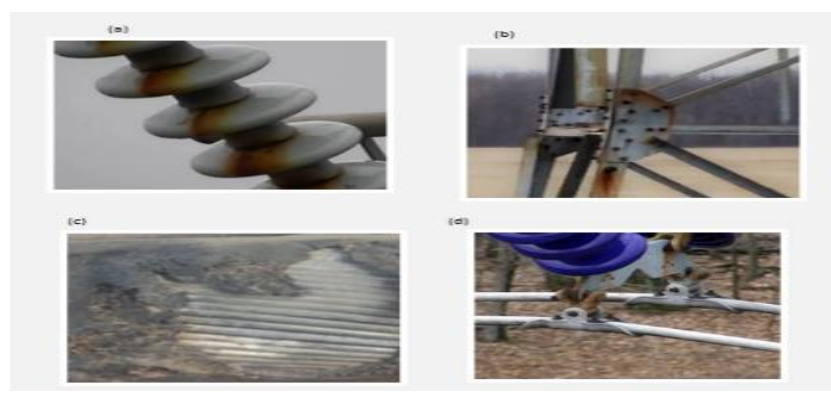

Fig. 5. Types Of Transmission Line Components Faults (A) Insulator Flashover (B) Transmission Tower Corrosion (C) Broken Power Line (D) Sag Adjusters Corrosion. 


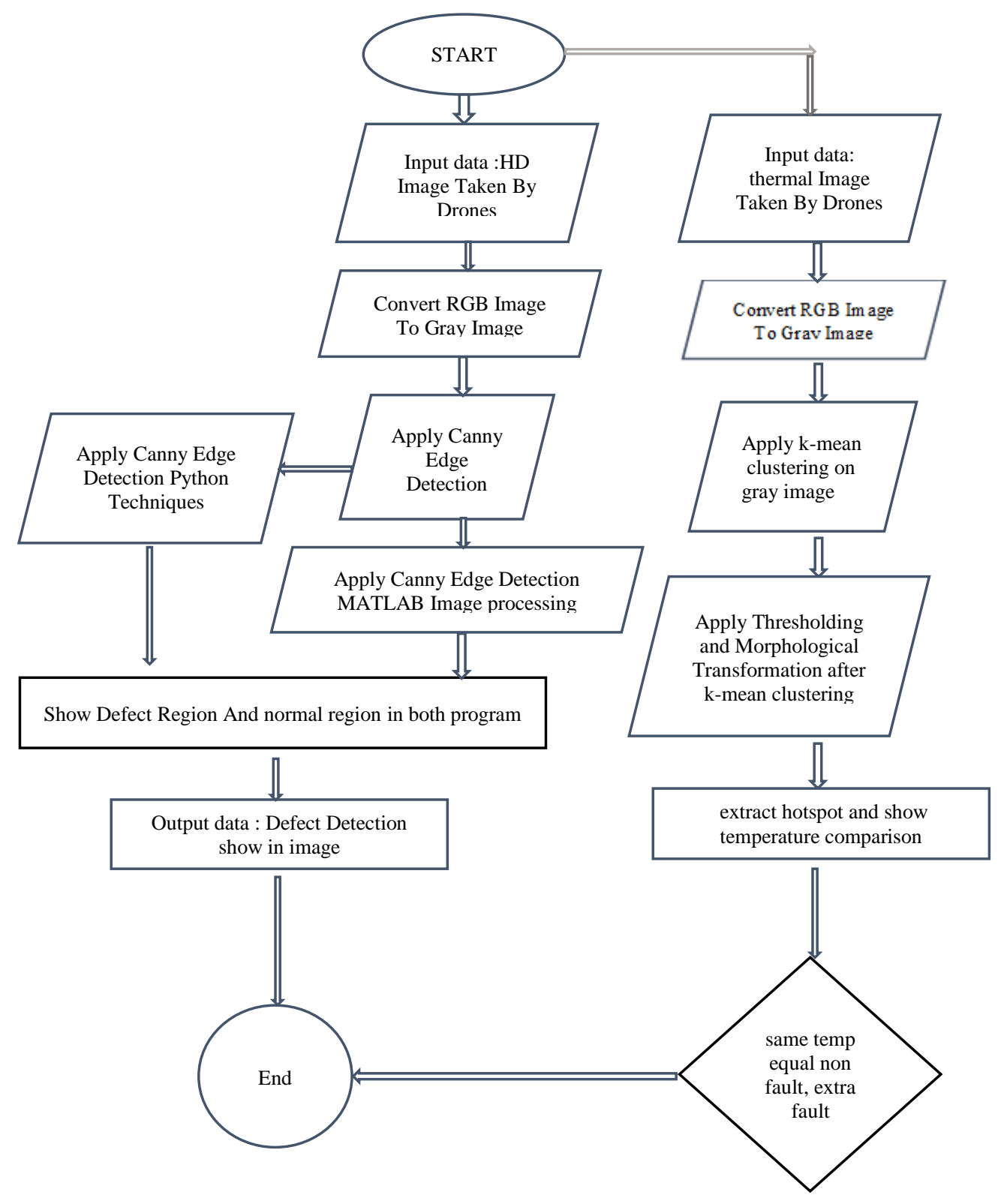

Fig.4 Methodology of The Proposed System. 


\subsection{Insulator Defect Detection}

\subsubsection{Defect A Broken Insulators Disk}

Broken insulators defect begins with small cracks on the ceramic disk Surface, because of thermal stress. Due to high winds and mechanical stress, these cracks grow over time, which completely breaks single disks apart. As shown in Fig. 6.

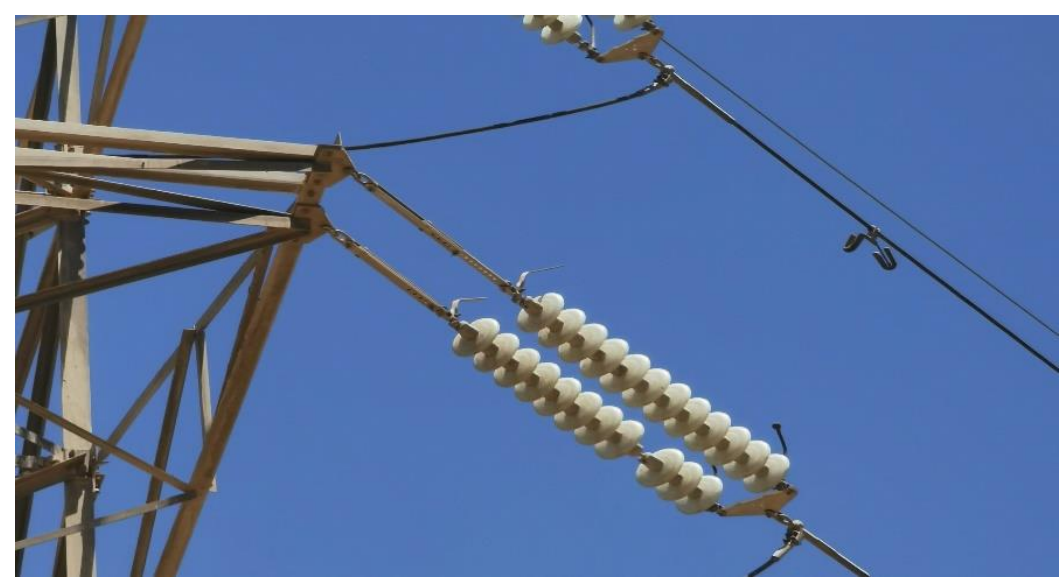

Fig. 6. Insulators broken shed.

\subsubsection{MATLAB Technique}
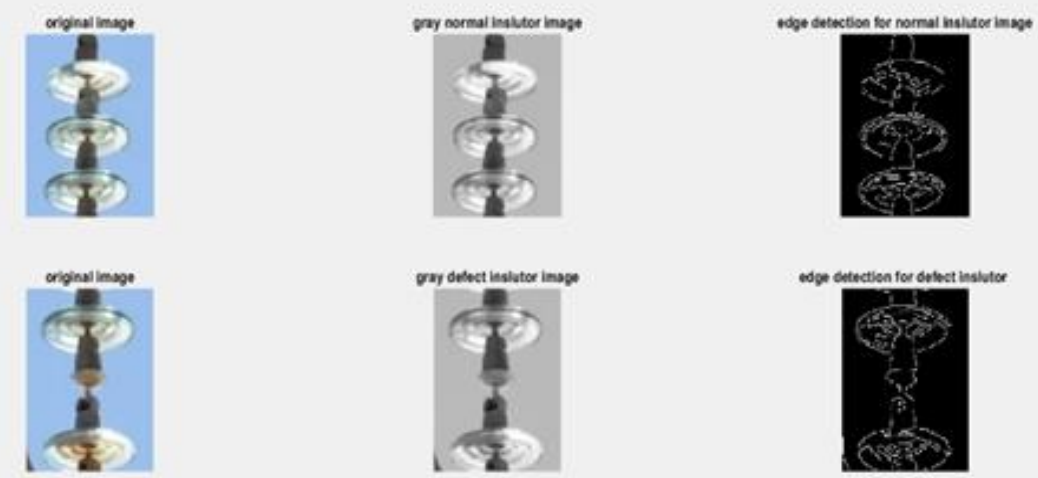

Fig. 7.image processing for normal and defect glass insulators 


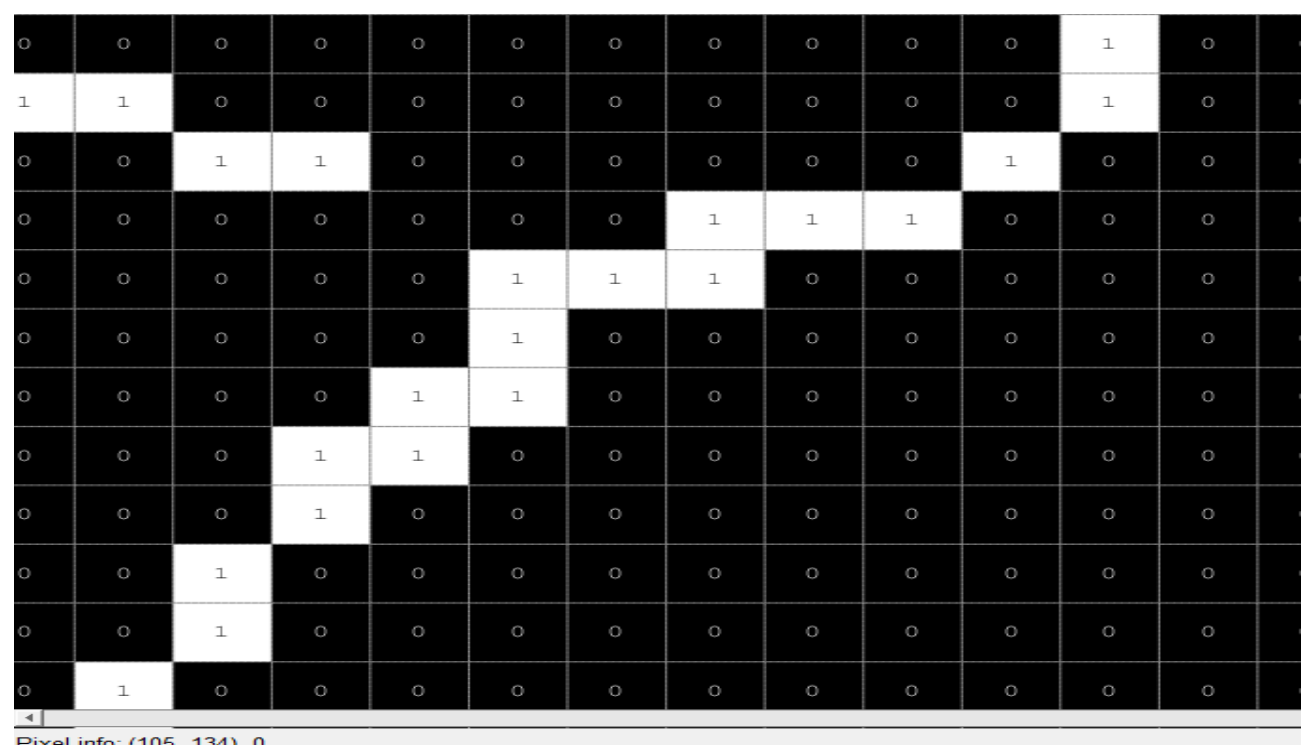

Fig. 8. Pixel region $(105,134)$ for normal glass insulator region.

\begin{tabular}{|c|c|c|c|c|c|c|c|c|c|c|c|c|c|}
\hline 0 & 0 & 0 & 0 & 0 & 0 & 0 & 0 & 0 & 0 & 0 & 0 & 1 & 0 \\
\hline 0 & 0 & 0 & 0 & 0 & 0 & 0 & 0 & 0 & 0 & 0 & 0 & 1 & 0 \\
\hline 0 & 0 & 0 & 0 & 0 & 0 & 0 & 0 & 0 & 0 & 0 & 0 & 1 & 0 \\
\hline 0 & 0 & 0 & 0 & 0 & 0 & 0 & 0 & 0 & 0 & 0 & 0 & 1 & 0 \\
\hline 0 & 0 & 0 & 0 & 0 & 0 & 0 & 0 & 0 & 0 & 0 & 0 & 1 & 0 \\
\hline 0 & 0 & 0 & 0 & 0 & 0 & 0 & 0 & 0 & 0 & 0 & 0 & 1 & 0 \\
\hline 0 & 0 & 0 & 0 & 0 & 0 & 0 & 0 & 0 & 0 & 0 & 1 & 1 & 0 \\
\hline 0 & 0 & 0 & 1 & 1 & 1 & 1 & 1 & 1 & 1 & 1 & 1 & 0 & 0 \\
\hline
\end{tabular}

Fig. 9. Pixel region $(105,134)$ for defect glass insulator region.

\subsubsection{Python Technique}



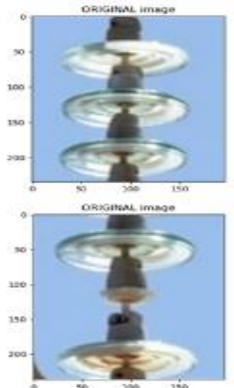

Fig.10. image processing for normal and defect glass insulators.

$\doteqdot \leftarrow \mathbb{E}$
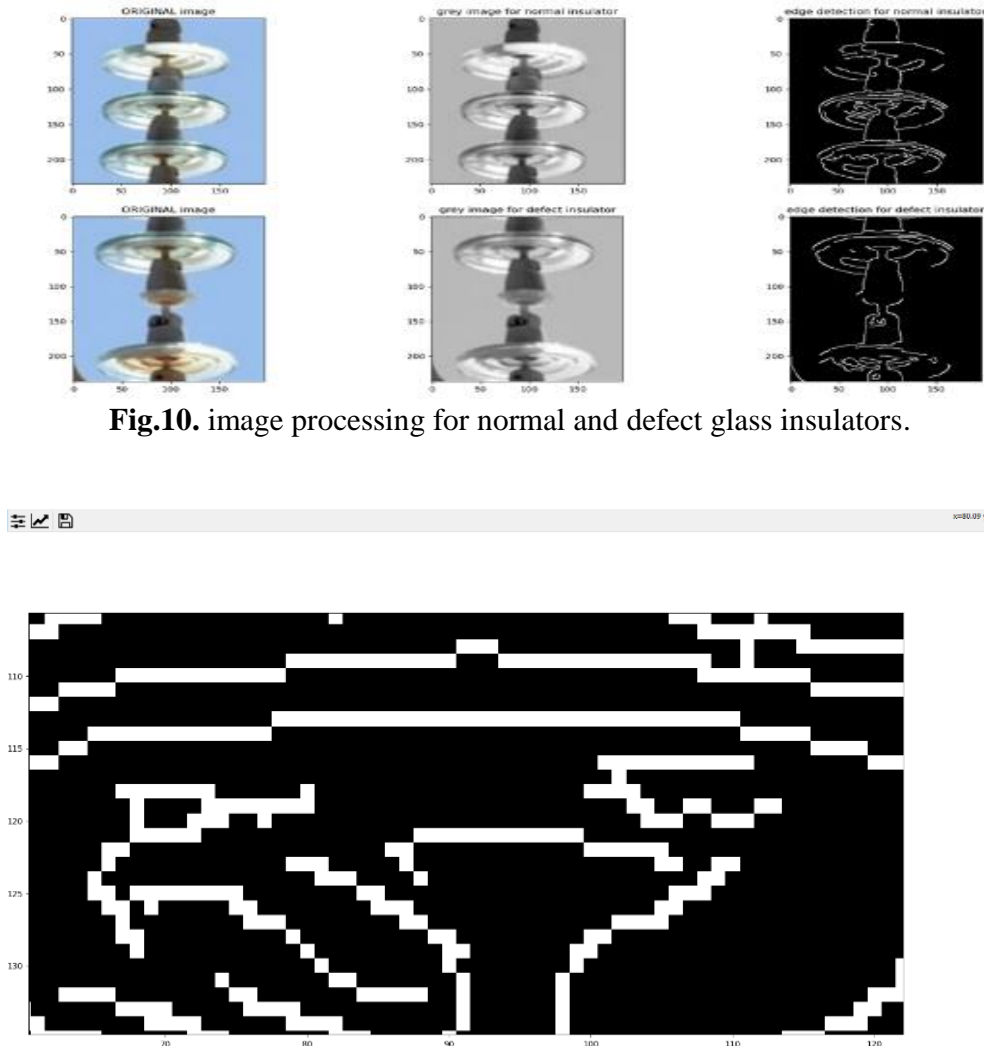

Fig. 11. Pixel region $(80.09,117.62)$ for normal glass insulator region. 曲

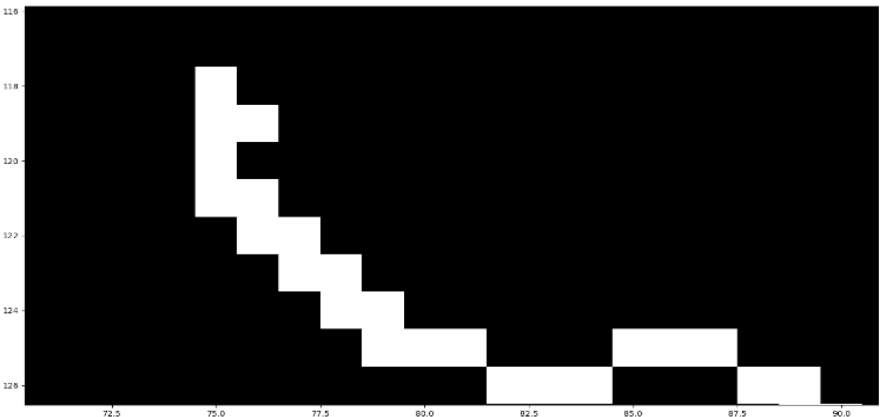

Fig. 12. Pixel region $(80.09,117.62)$ for defect glass insulator region.

\subsubsection{Defect A Flashover Insulators}


In a flashover case, pollution like the lightning, the bird, icing, and Desert is the most important factor. One of the most serious high voltage energy transmission matters is the flashover of polluted high voltage insulators.

\subsubsection{MATLAB Technique}

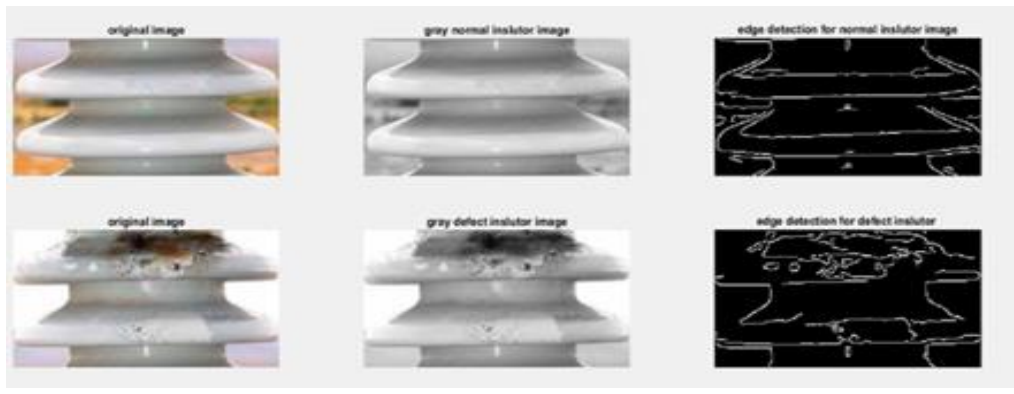

Fig.13. image processing for normal and defect porcelain insulators

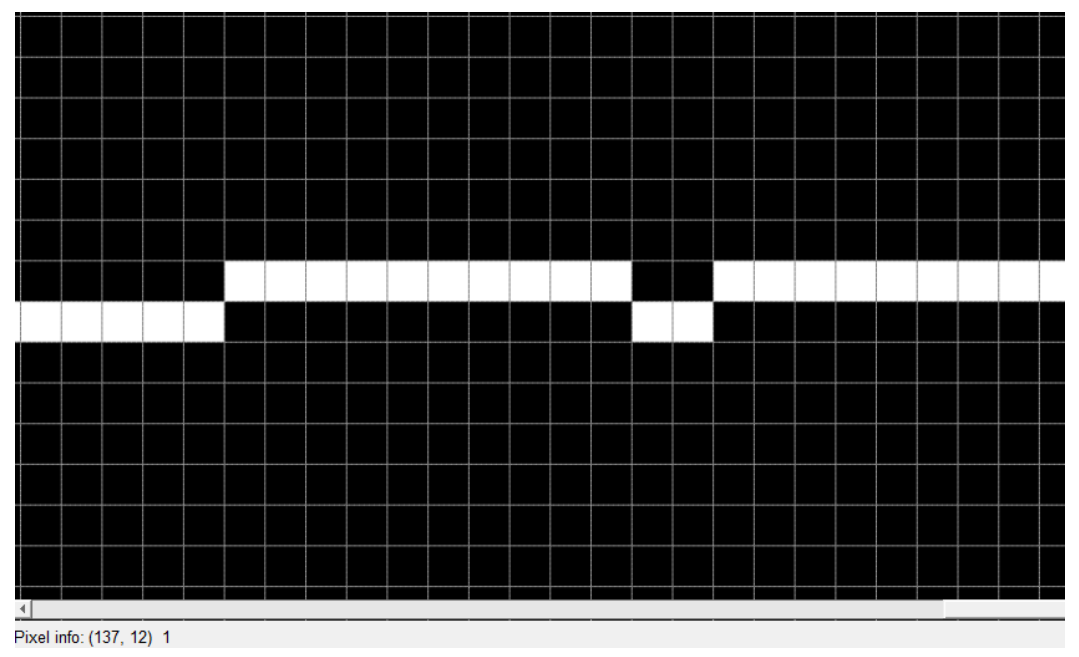

Fig.14. Pixel region $(137,12)$ for normal porcelain insulator region. 


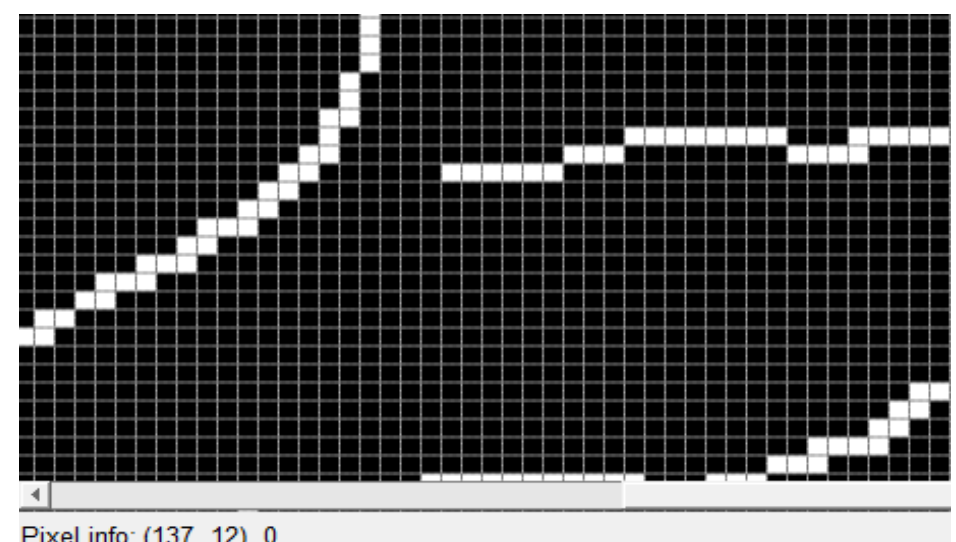

Fig.15. Pixel region $(137,12)$ for defect porcelain insulator region

\subsubsection{Python Technique}

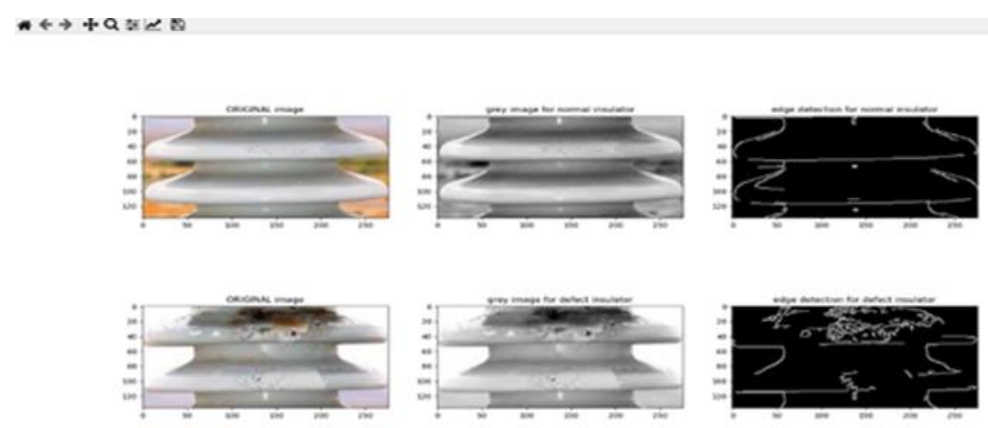

Fig.16. image processing for normal and defect porcelain insulators $\doteqdot \sim ⿴ 囗 十$

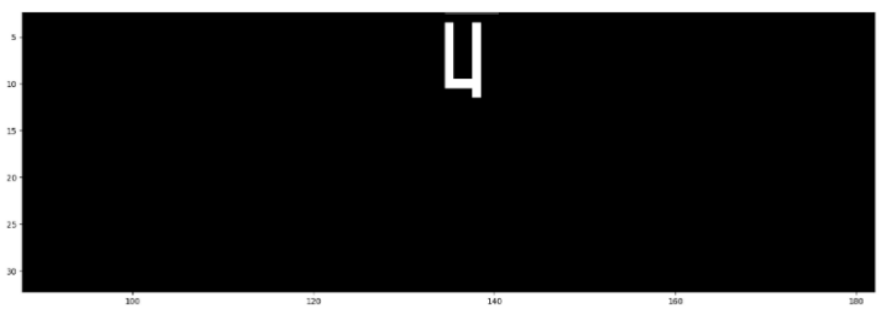

Fig. 17. Pixel region $(135.03,10.09)$ for normal porcelain insulator region. 


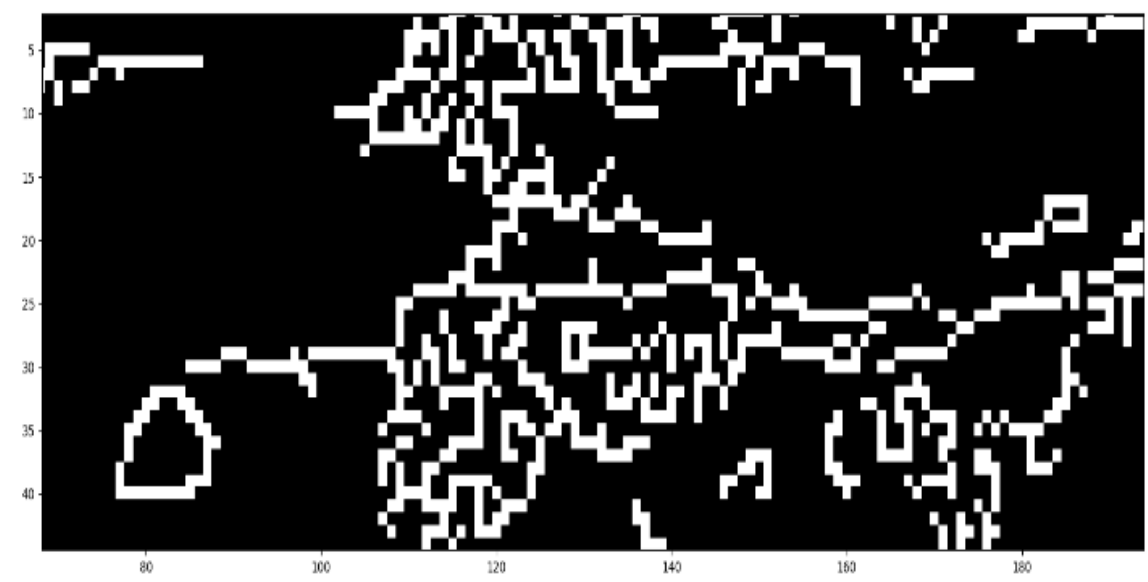

Fig.18. Pixel region $(135.03,10.09)$ for defect porcelain insulator region

\subsection{Discuss the Results}

In this paper, the algorithm is designed to process HD images of power line components captured by drones. That should be filtered by canny edge detection. Defect insulators were used by canny edge detection to diagnose the finding of rough and soft areas in python and MATLAB and discuss the results in both programs to find the best results. Canny edge detection is a multistage algorithm that includes the first step is denoising the image with a gaussian filter then the smooth image is filtered with a Sobel kernel and then a full image scan is done to remove any unwanted pixels which may not be from the edge. The final step hysteresis thresholding this stage decides which edges are true edges and which are not. Cranny's method is not easily disturbed by noise and true weak edges can be revealed. The advantage is that two different thresholds are used to detect the strong edge and the weak edge respectively. When the weak edge and the strong edge are connected, the weak edge is included in the output image. Therefore, it is easy to specify the upper and lower threshold values to display changes in the image such as select original object editors, delete edges of noise effect, and adjust the lighting intensity. the result is more accurate in python, although it allows setting a factor a threshold of less than 1 in MATLAB. Matplotlib library in python for display pixel areas is more accurate than using MATLAB's image tools.

\section{Conclusion}

This paper focuses on texture features that can detect the external structure of an image. Although the ordinary insulator and the defect insulator have different values for the property 
of the fabric. Edge detection algorithm used in matplotlib plot order in python and image order tool in MATLAB to manually select important pixels in images. The pixels estimate the $\mathrm{x}$-axis and $y$-axis in MATLAB as follows $(105,34)$ is equal to zero indicating that the region has no defect or equal to one which indicates a defective region as shown in fig. 7.8. The pixel area in python (80.09117.62) applied the same rule as shown in fig. 11,12. The value of the area of the ceramic insulator in MATLAB $(137,12)$ was found to be 0 for normal insulators and one for defect, as shown in fig. 14.15. While it is found in python $(138.06,24.97)$ as shown in fig. 17.18. These pixel areas appear in black and white based on the edges that can be found in the image. White indicates the large number of raised edges represented by 1 or 255 , while black indicates the smooth area represented by 0 . In this method, an insulator representing the normal sample and the defect sample must be used for the comparison value to explain the difference between them. Also, in this less expensive method is used to treat the thermal image by obtaining a hot spot from a faulty insulator or another compound from the power line. As a result, such a defect in the insulator may lead to breakdown due to a malfunction in the power line network and will be the cause of stopping the power supply to the consumer.

\section{References}

[1] X. Xie, Z. Liu, C. Xu, and Y. Zhang, "A multiple sensors platform method for power line inspection based on a large unmanned helicopter," Sensors (Switzerland), vol. 17, no. 6, pp. 117, 2017, DOI: 10.3390/s17061222.

[2] L. F. Luque-Vega, B. Castillo-Toledo, A. Loukianov, and L. E. Gonzalez-Jimenez, "Power line inspection via an unmanned aerial system based on the quadrotor helicopter," in MELECON 2014-2014 17th IEEE Mediterranean electrotechnical conference, 2014, pp. 393-397.

[3] L. Guo, Y. Liao, H. Yao, J. Chen, and M. Wang, "An Electrical Insulator Defects Detection Method Combined Human Receptive Field Model,” J. Control Sci. Eng., vol. 2018, 2018, doi: $10.1155 / 2018 / 2371825$.

[4] J. Wu, W. Fei, and Q. Li, “An integrated measure and location method based on airborne 2d laser scanning sensor for UAV's power line inspection," Proc. - 2013 5th Conf. Meas. Technol. Mechatronics Autom. ICMTMA 2013, pp. 213-217, 2013, doi: 10.1109/ICMTMA.2013.58.

[5] Y. Zhai, R. Chen, Q. Yang, X. Li, and Z. Zhao, "Insulator fault detection based on spatial morphological features of aerial images," IEEE Access, vol. 6, pp. 35316-35326, 2018, doi: 10.1109/ACCESS.2018.2846293.

[6] M. Pech, J. Vrchota, and J. Bednář, "Predictive maintenance and intelligent sensors in smart factory: Review," Sensors, vol. 21, no. 4, pp. 1-39, 2021, doi: 10.3390/s21041470.

[7] P. S. F. Sheron, K. P. Sridhar, S. Baskar, and P. M. Shakeel, "Projection-dependent input processing for 3D object recognition in human robot interaction systems," Image Vis. Comput. vol. 106, p. 104089, 2021, doi: 10.1016/j.imavis.2020.104089.

[8] Z. A. Siddiqui and U. Park, "A Drone Based Transmission Line Components Inspection System with Deep Learning Technique," Energies, vol. 13, no. 13, 2020, doi: 10.3390/en13133348.

[9] J. I. Larrauri, G. Sorrosal, and M. Gonzalez, "Automatic system for overhead power line inspection using an Unmanned Aerial Vehicle - RELIFO project," 2013 International Conference on Unmanned Aircraft Systems, ICUAS 2013 - Conference Proceedings. pp. 244-252, 2013, doi: 10.1109/ICUAS.2013.6564696.

[10] P. Leitao, P. Vrba, and T. Strasser, "Multi-agent systems as automation platform for intelligent energy systems," IECON Proc. (Industrial Electron. Conf., pp. 66-71, 2013, doi: 10.1109/IECON.2013.6699113.

[11] A. Baba, "A new design of a flying robot, with advanced computer vision techniques to perform self-maintenance of smart grids," J. King Saud Univ. - Comput. Inf. Sci., no. xxxx, 2020, doi: 10.1016/j.jksuci.2020.07.009.

[12] M. Korki, N. D. Shankar, R. N. Shah, S. M. Waseem, and S. Hodges, "Automatic Fault Detection 
of Power Lines using Unmanned Aerial Vehicle (UAV)," 2019 1st International Conference on Unmanned Vehicle Systems-Oman, UVS 2019. 2019, doi: 10.1109/UVS.2019.8658283.

[13] "infrared-and-ultraviolet-2002 (2).pdf.".

[14] B. Jalil, G. R. Leone, M. Martinelli, D. Moroni, M. A. Pascali, and A. Berton, "Fault detection in power equipment via an unmanned aerial system using multi modal data," Sensors, vol. 19, no. 13, p. 3014, 2019.

[15] F. Gao et al., "Recognition of insulator explosion based on deep learning," 2016 13th International Computer Conference on Wavelet Active Media Technology and Information Processing, ICCWAMTIP 2017, vol. 2018-February. pp. 79-82, 2017, doi: 10.1109/ICCWAMTIP.2017.8301453. 